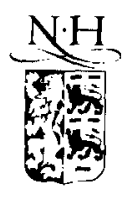

FISSFVIF:R
2 March 1995

PHYSICS LETTERS B

Physics Letters B 346 ( 1995) 190-198

\title{
Measurement of energetic single-photon production at LEP
}

\section{L3 Collaboration}

M. Acciarri ${ }^{z}$, A. Adam ${ }^{\text {aq }}$, O. Adriani ${ }^{p}$, M. Aguilar-Benitez ${ }^{y}$, S. Ahlen ${ }^{j}$, J. Alcaraz $^{y}$, A. Aloisio $^{\text {ab }}$, G. Alverson ${ }^{\text {k }}$, M.G. Alviggi ${ }^{\text {ab }}$, G. Ambrosi ag, Q. An ${ }^{\mathrm{r}}$, H. Anderhub ${ }^{\text {at }}$, A.L. Anderson ${ }^{\circ}$, V.P. Andreev ${ }^{\text {ak }}$, T. Angelescu ${ }^{\ell}$, L. Antonov an , D. Antreasyan ${ }^{\text {h }}$, G. Alkhazov ak, P. Arce ${ }^{y}$, A. Arefiev ${ }^{\text {aa }}$, T. Azemoon ${ }^{c}$, T. Aziz ${ }^{\mathrm{i}}$, P.V.K.S. Baba ${ }^{\mathrm{r}}$, P. Bagnaia ${ }^{\mathrm{aj}, \mathrm{q}}$, J.A. Bakken ${ }^{\text {ai }}$, L. Baksay ${ }^{\text {ap }, \text { R.C. Ball }}{ }^{\text {c }}$, S. Banerjee ${ }^{\mathrm{i}}, \mathrm{K}$ Banicz $^{\text {aq }}$, R. Barillère ${ }^{\mathrm{q}}$, L. Barone $^{\text {aj }}$, A. Baschirotto ${ }^{z}$, M. Basile ${ }^{\text {h }}$, R. Battiston ag ${ }^{\text {ag }}$ A. Bay ${ }^{v}$, F. Becattini ${ }^{\text {P }}$, U. Becker ${ }^{\circ}$, F. Behner ${ }^{\text {at }}$, Gy.L. Bencze ${ }^{\mathrm{m}}$, J. Berdugo ${ }^{\mathrm{y}}$, P. Berges ${ }^{\circ}$, B. Bertucci ${ }^{\mathrm{q}}$, B.L. Betev ${ }^{\text {an, at }}$, M. Biasini ${ }^{\text {ag }}$, A. Biland ${ }^{\text {at }}$, G.M. Bilei ${ }^{\text {ag }}$, R. Bizzarri ${ }^{\text {aj }}$, J.J. Blaising ${ }^{\text {d }}$, G.J. Bobbink ${ }^{\text {q.b }}$, R. Bock ${ }^{\text {a }}$, A. Böhm ${ }^{\text {a }}$, B. Borgia ${ }^{\text {aj }}$, A. Boucham ${ }^{d}$, D. Bourilkov ${ }^{\text {at }}$, M. Bourquin ${ }^{\text {s }}$, D. Boutigny ${ }^{q}$, B. Bouwens ${ }^{b}$,

E. Brambilla ${ }^{\circ}$, J.G. Branson ${ }^{\text {a }}$, V. Brigljevic ${ }^{\text {at }}$, I.C. Brock ${ }^{\text {ah }}$, M. Brooks ${ }^{\text {w }}$, A. Bujak ${ }^{\text {aq }}$, J.D. Burger ${ }^{\circ}$, W.J. Burger ${ }^{\mathrm{s}}$, C. Burgos ${ }^{\mathrm{y}}$, J. Busenitz $^{\text {ap }}$, A. Buytenhuijs ${ }^{\text {ad }}$, A. Bykov ${ }^{\text {ak }}$,

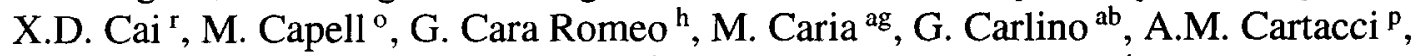
J. Casaus ${ }^{y}$, R. Castello ${ }^{z}$, N. Cavallo ${ }^{\text {ab }}$, M. Cerrada ${ }^{y}$, F. Cesaroni ${ }^{\text {aj }}$, M. Chamizo ${ }^{y}$, Y.H. Chang av, U.K. Chaturvedi ${ }^{\mathrm{r}}$, M. Chemarin ${ }^{\mathrm{x}}$, A. Chen ${ }^{\text {av }}$, C. Chen ${ }^{\mathrm{f}}$, G. Chen ${ }^{\mathrm{f}}$, G.M. Chen ${ }^{\mathrm{f}}$, H.F. Chen ${ }^{t}$, H.S. Chen ${ }^{f}$, M. Chen ${ }^{\circ}$, G. Chiefari ${ }^{\text {ab }}$, C.Y. Chien ${ }^{\mathrm{e}}$, M.T. Choi ${ }^{\text {ao }}$, S. Chung ${ }^{\circ}$, L. Cifarelli ${ }^{\text {h}}$, F. Cindolo ${ }^{\text {h }}$, C. Civinini ${ }^{\text {p }}$, I. Clare ${ }^{\text {o }}$, R. Clare ${ }^{\text {o }}$, T.E. Coan ${ }^{\text {w }}$, H.O. Cohn ${ }^{\text {ac }}$, G. Coignet ${ }^{\mathrm{d}}$, N. Colino ${ }^{\mathrm{q}}$, V. Commichau ${ }^{\mathrm{a}}$, S. Costantini ${ }^{\text {aj, }}$, F. Cotorobai ${ }^{\ell}$, B. de la Cruz ${ }^{\mathrm{y}}$, X.T. Cui ${ }^{r}$, X.Y. Cui ${ }^{r}$, T.S. Dai ${ }^{\circ}$, R. D'Alessandro ${ }^{p}$, R. de Asmundis ${ }^{\text {ab }}$, A. Degré $^{d}$, K. Deiters ${ }^{\text {ar }}$, E. Dénes ${ }^{m}$, P. Denes ${ }^{\text {ai }}$, F. DeNotaristefani ${ }^{\text {aj }}$, D. DiBitonto ${ }^{\text {ap }}$, M. Diemoz $^{\text {aj }}$, H.R. Dimitrov ${ }^{\text {an }}$, C. Dionisi ${ }^{\text {aj }}$, M. Dittmar ${ }^{\text {at }}$, I. Dorne ${ }^{d}$, M.T. Dova ${ }^{\text {r,3 }}$, E. Drago ${ }^{\text {ab }}$, D. Duchesneau ${ }^{\text {q }}$, F. Duhem ${ }^{d}$, P. Duinker ${ }^{b}$, I. Duran ${ }^{a m}$, S. Dutta ${ }^{\text {i }}$, S. Easo ${ }^{\text {ag }}$, H. El Mamouni ${ }^{\text {, }}$, A. Engler ${ }^{\text {ah }}$, F.J. Eppling ${ }^{\circ}$, F.C. Erné ${ }^{b, q}$, P. Extermann ${ }^{s}$, R. Fabbretti ${ }^{\text {ar }}$, M. Fabre ${ }^{\text {ar }}$, S. Falciano ${ }^{\text {aj }}$, A. Favara ${ }^{p}$, J. Fay ${ }^{x}$, M. Felcini ${ }^{\text {at }}$, T. Ferguson ${ }^{\text {ah }}$, D. Fernandez ${ }^{y}$, G. Fernandez ${ }^{y}$, F. Ferroni ${ }^{\text {aj }}$, H. Fesefeldt ${ }^{a}$, E. Fiandrini ${ }^{\text {ag }}$, J.H. Field ${ }^{\text {s }}$, F. Filthaut ${ }^{\text {ad }}$, P.H. Fisher ${ }^{\text {e }}$, G. Forconi ${ }^{\circ}$, L. Fredj ${ }^{\mathrm{s}}$, K. Freudenreich ${ }^{\text {at }}$, M. Gailloud ${ }^{\mathrm{v}}$, Yu. Galaktionov ${ }^{\mathrm{a} a, 0}$, E. Gallo ${ }^{\mathrm{p}}$, S.N. Ganguli ${ }^{\mathrm{i}}$, P. Garcia-Abia ${ }^{y}$, S.S. Gau ${ }^{k}$, S. Gentile ${ }^{\text {aj, J. Gerald }}{ }^{\mathrm{e}}$, N. Gheordanescu ${ }^{\ell}$, S. Giagu ${ }^{\text {aj, }}$ S. Goldfarb ${ }^{v}$, J. Goldstein ${ }^{\mathrm{j}}$, Z.F. Gong ${ }^{\mathrm{t}}$, E. Gonzalez ${ }^{y}$, A. Gougas ${ }^{\mathrm{e}}$, D. Goujon ${ }^{\mathrm{s}}$, G. Gratta af M.W. Gruenewald ${ }^{\mathrm{g}}$, C. Gu ${ }^{\mathrm{r}}$, M. Guanziroli ${ }^{\mathrm{r}}$, V.K. Gupta ${ }^{\mathrm{a}}$, A. Gurtu ${ }^{\mathrm{i}}$, H.R. Gustafson ${ }^{\mathrm{c}}$,

L.J. Gutay ${ }^{\text {aq }}$, B. Hartmann ${ }^{\text {a }}$, A. Hasan ${ }^{\text {ac }}$, D. Hauschildt ${ }^{\text {b }}$, J.T. He ${ }^{\text {f }}$, T. Hebbeker ${ }^{\mathrm{g}}$,

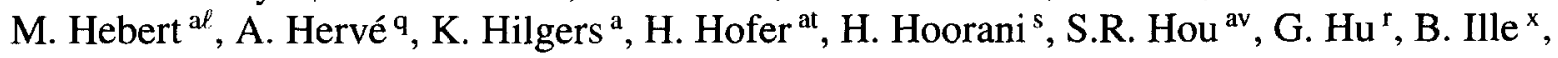


M.M. Ilyas ${ }^{\mathrm{r}}$, V. Innocente ${ }^{\mathrm{q}}$, H. Janssen ${ }^{\mathrm{d}}$, B.N. Jin ${ }^{\mathrm{f}}$, L.W. Jones ${ }^{\mathrm{c}}$, P. de Jong ${ }^{\circ}$, I. Josa-Mutuberria ${ }^{q}$, A. Kasser ${ }^{\text {v }}$, R.A. Khan ${ }^{\mathrm{r}}$, Yu. Kamyshkov ${ }^{\text {ae }}$, P. Kapinos ${ }^{\text {as }}$, J.S. Kapustinsky w, Y. Karyotakis ${ }^{q}$, M. Kaur ${ }^{\text {r }}$, S. Khokhar ${ }^{r}$, M.N. Kienzle-Focacci ${ }^{\text {s }}$, D. Kim ${ }^{\mathrm{e}}$, J.K. Kim ${ }^{\text {ac }}$, S.C. Kim $^{\text {ac }}$, Y.G. Kim ${ }^{\text {ac }}$, W.W. Kinnison ${ }^{\text {w }}$, A. Kirkby ${ }^{\text {af }}$, D. Kirkby ${ }^{\text {af }}$, J. Kirkby ${ }^{q}$, S. Kirsch ${ }^{\text {as }}$, W. Kittel ${ }^{\text {ad }}$, A. Klimentov ${ }^{\text {o,aa }}$, A.C. König ${ }^{\text {ad }}$, E. Koffeman ${ }^{\text {b }}$, O. Kornadt ${ }^{\text {a }}$, V. Koutsenko ${ }^{\text {o,aa }}$, A. Koulbardis ${ }^{\text {ak }}$, R.W. Kraemer ${ }^{\text {ah }}$, T. Kramer ${ }^{\circ}$, V.R. Krastev an,ag, W. Krenz ${ }^{a}$, H. Kuijten ${ }^{\text {ad }}$, A. Kunin ${ }^{\text {o,aa }}$, P. Ladron de Guevara ${ }^{\mathrm{y}, \mathrm{q}}$, G. Landi $^{\mathrm{p}}$, S. Lanzano $^{\mathrm{ab}, 4}{ }^{\text {, }}$ P. Laurikainen ${ }^{\mathrm{u}}$, A. Lebedev ${ }^{\circ}$, P. Lebrun ${ }^{\mathrm{x}}$, P. Lecomte ${ }^{\text {at }}$, P. Lecoq ${ }^{\mathrm{q}}$, P. Le Coultre ${ }^{\text {at }}$, D.M. Lee ${ }^{w}$, J.S. Lee ${ }^{\text {ao }}$, K.Y. Lee ${ }^{\text {ao }}$, I. Leedom ${ }^{k}$, C. Leggett ${ }^{c}$, J.M. Le Goff ${ }^{q}$, R. Leiste ${ }^{\text {as }}$, M. Lenti ${ }^{\text {p }}$, E. Leonardi aj, P. Levtchenko ${ }^{\text {ak }}$, C. Li ${ }^{\text {t,r }}$, E. Lieb ${ }^{\text {as }}$, W.T. Lin ${ }^{\text {av }}$, F.L. Linde ${ }^{\text {b }}$, B. Lindemann ${ }^{a}$, L. Lista ${ }^{\text {ab }}$, Y. Liu ${ }^{r}$, W. Lohmann ${ }^{\text {as }}$, E. Longo ${ }^{\text {aj }}$, W. Lu ${ }^{\text {af }}$, Y.S. Lu ${ }^{\text {f }}$, J.M. Lubbers ${ }^{\text {q }}$, K. Lübelsmeyer ${ }^{\text {a }}$, C. Luci ${ }^{\text {aj }}$, D. Luckey ${ }^{\circ}$, L. Ludovici ${ }^{\text {aj }, ~ L . ~ L u m i n a r i ~}{ }^{\text {aj }}$, W. Lustermann ${ }^{\text {ar }}$, W.G. Ma ${ }^{t}$, M. MacDermott ${ }^{\text {at }}$, M. Maity ${ }^{\mathrm{i}}$, L. Malgeri ${ }^{\text {aj }}$, R. Malik ${ }^{\mathrm{r}}$, A. Malinin ${ }^{\text {aa }}$, C. Maña ${ }^{y}$, S. Mangla ${ }^{i}$, M. Maolinbay ${ }^{\text {at }}$, P. Marchesini ${ }^{\text {at }}$, A. Marin ${ }^{j}$, J.P. Martin ${ }^{x}$, F. Marzano aj, G.G.G. Massaro ${ }^{b}$, K. Mazumdar ${ }^{i}$, T. McMahon ${ }^{\text {aq }}$, D. McNally ${ }^{a \ell}$, S. Mele ${ }^{\text {ab }}$, M. Merk ${ }^{\text {ah }}$, L. Merola ${ }^{a b}$, M. Meschini ${ }^{p}$, W.J. Metzger ${ }^{a d}$, Y. Mi $^{v}$, A. Mihul ${ }^{\ell}$, G.B. Mills ${ }^{\text {w }}$, Y. Mir ${ }^{r}$, G. Mirabelli ${ }^{\text {aj }}$, J. Mnich ${ }^{a}$, M. Möller ${ }^{a}$, V. Monaco ${ }^{\text {aj }}$, B. Monteleoni ${ }^{p}$, R. Morand ${ }^{d}$, S. Morganti ${ }^{\text {aj, }}$, N.E. Moulai ${ }^{r}$, R. Mount ${ }^{\text {af }}$, S. Müller ${ }^{a}$, E. Nagy ${ }^{m}$, M. Napolitano ${ }^{\text {ab }}$, F. Nessi-Tedaldi ${ }^{\text {at }}$, H. Newman ${ }^{\text {af }}$, M.A. Niaz ${ }^{\mathrm{r}}$, A. Nippe ${ }^{\text {a }}$, H. Nowak ${ }^{\text {as }}$, G. Organtini ${ }^{\text {aj }}$, R. Ostonen ${ }^{\text {}}$, D. Pandoulas ${ }^{a}$, S. Paoletti ${ }^{\text {aj }}$, P. Paolucci ${ }^{\text {ab }}$, G. Pascale ${ }^{\text {aj }}$, G. Passaleva ${ }^{\text {p,ag }}$, S. Patricelli ${ }^{\text {ab }}$, T. Paul ${ }^{\text {e }}$, M. Pauluzzi ${ }^{\text {ag }}$, C. Paus ${ }^{\text {a }}$, F. Pauss ${ }^{\text {at }}$, Y.J. Pei ${ }^{\text {a }}$, S. Pensotti ${ }^{\mathrm{z}}$, D. Perret-Gallix ${ }^{\mathrm{d}}$, A. Pevsner ${ }^{\mathrm{e}}$, D. Piccolo ${ }^{\mathrm{ab}}$, M. Pieri ${ }^{\mathrm{p}}$, J.C. Pinto $^{\text {ah }}$, P.A. Piroué ${ }^{\text {ai }}$, E. Pistolesi ${ }^{\text {p }}$, F. Plasil ${ }^{\text {ae }}$, V. Plyaskin ${ }^{\text {aa }}$, M. Pohl $^{\text {at }}$, V. Pojidaev ${ }^{\text {aa, }}$, , H. Postema ${ }^{\circ}$,

N. Produit ${ }^{\text {s }}$, J.M. Qian ${ }^{\text {c }}$, K.N. Qureshi ${ }^{\mathrm{r}}$, R. Raghavan ${ }^{\mathrm{i}}$, G. Rahal-Callot ${ }^{\text {at }}$, P.G. Rancoita ${ }^{\mathrm{z}}$, M. Rattaggi ${ }^{\text {z }}$, G. Raven ${ }^{\text {b }}$, P. Razis ${ }^{\text {ac }}$, K. Read ${ }^{\text {ae }}$, M. Redaelli ${ }^{7}$, D. Ren ${ }^{\text {at }}$, Z. Ren ${ }^{r}$, M. Rescigno ${ }^{\text {aj }}$, S. Reucroft ${ }^{k}$, A. Ricker ${ }^{a}$, S. Riemann ${ }^{\text {as }}$, B.C. Riemers ${ }^{\text {aq }}$, K. Riles ${ }^{c}$, O. Rind ${ }^{\text {c }}$, H.A. Rizvi ${ }^{r}$, S. Ro ${ }^{\text {ao }}$, A. Robohm ${ }^{\text {at }}$, F.J. Rodriguez ${ }^{y}$, B.P. Roe ${ }^{c}$, M. Röhner ${ }^{\text {a }}$, S. Röhner ${ }^{a}$, L. Romero ${ }^{y}$, S. Rosier-Lees ${ }^{d}$, R. Rosmalen ${ }^{\text {ad }}$, Ph. Rosselet ${ }^{\text {v }}$, W. van Rossum ${ }^{b}$, S. Roth ${ }^{a}$, A. Rubbia ${ }^{\circ}$, J.A. Rubio ${ }^{q}$, H. Rykaczewski ${ }^{\text {at }}$, J. Salicio ${ }^{\text {q }}$, J.M. Salicio ${ }^{y}$, E. Sanchez ${ }^{y}$, G.S. Sanders ${ }^{\text {w }}$, A. Santocchia ${ }^{\text {ag }}$, M.E. Sarakinos ${ }^{\text {aq }}$, S. Sarkar ${ }^{\mathrm{i}}$, G. Sartorelli ${ }^{\mathrm{r}}$, M. Sassowsky ${ }^{\text {a }}$, G. Sauvage ${ }^{d}$, C. Schäfer ${ }^{a}$, V. Schegelsky ${ }^{\text {ak }}$, D. Schmitz ${ }^{a}$, P. Schmitz ${ }^{a}$, M. Schneegans ${ }^{d}$, N. Scholz ${ }^{\text {at }}$, H. Schopper ${ }^{\text {au }}$, D.J. Schotanus ${ }^{\text {ad }}$, S. Shotkin ${ }^{\circ}$, H.J. Schreiber ${ }^{\text {as }}$, J. Shukla ${ }^{\text {w }}$, R. Schulte ${ }^{a}$, K. Schultze ${ }^{\text {a }}$ J. Schwenke ${ }^{a}$, G. Schwering ${ }^{a}$, C. Sciacca ${ }^{\text {ab }}$, R. Sehgal ${ }^{r}$, P.G. Seiler ${ }^{\text {ar }}$, J.C. Sens ${ }^{\text {q,b }}$, L. Servoli ${ }^{\text {ag }}$, I. Sheer ${ }^{a \ell}$, S. Shevchenko ${ }^{\text {af }}$, X.R. Shi ${ }^{\text {af }}$, E. Shumilov ${ }^{\text {aa }}$, V. Shoutko ${ }^{\text {aa }}$, D. Son ${ }^{\text {ao }}$, A. Sopczak ${ }^{\text {q }}$, V. Soulimov ${ }^{\text {ab }}$, C. Spartiotis ${ }^{\text {, }}$, T. Spickermann ${ }^{\text {a }}$, P. Spillantini ${ }^{p}$, M. Steuer ${ }^{\circ}$, D.P. Stickland ${ }^{\text {ai }}$, F. Sticozzi ${ }^{\circ}$, H. Stone $^{\text {ai }}$, K. Strauch ${ }^{n}$, K. Sudhakar ${ }^{i}$, G. Sultanov ${ }^{\text {r }}$, L.Z. Sun ${ }^{\text {t,r }}$, G.F. Susinno ${ }^{\text {s }}$, H. Suter ${ }^{\text {at }}$, J.D. Swain ${ }^{\mathrm{r}}$, A.A. Syed ${ }^{\text {ad }}$, X.W. Tang ${ }^{f}$, L. Taylor ${ }^{k}$, R. Timellini ${ }^{\text {h}}$, Samuel C.C. Ting ${ }^{\circ}$, S.M. Ting ${ }^{\circ}$, O. Toker ${ }^{\text {ag }}$, M. Tonutti ${ }^{\mathrm{a}}$, S.C. Tonwar ${ }^{\mathrm{i}}$, J. Tóth ${ }^{\mathrm{m}}$, G. Trowitzsch ${ }^{\text {as }}$, A. Tsaregorodtsev ${ }^{\mathrm{ak}}$, G. Tsipolitis ${ }^{\text {ah }}$, C. Tully ai ${ }^{\text {a }}$ J. Ulbricht ${ }^{\text {at }}$, L. Urbán ${ }^{\mathrm{m}}$, U. Uwer ${ }^{\mathrm{a}}$, E. Valente ${ }^{\text {aj }}$, 
R.T. Van de Walle ad, I. Vetlitsky ${ }^{\text {aa }}$, G. Viertel ${ }^{\text {at }}$, P. Vikas ${ }^{r}$, U. Vikas ${ }^{r}$, M. Vivargent ${ }^{d}$, H. Vogel ${ }^{\text {ah }}, H$. Vogt ${ }^{\text {as }}$, I. Vorobiev $^{\text {n,aa }}$, A.A. Vorobyov ${ }^{\text {ak }}$, An.A. Vorobyov ${ }^{\text {ak }}$, L. Vuilleumier ${ }^{\mathrm{v}}$, M. Wadhwa ${ }^{y}$, W. Wallraff ${ }^{a}$, J.C. Wang ${ }^{\circ}$, X.L. Wang ${ }^{t}$, Y.F. Wang ${ }^{\circ}$, Z.M. Wang ${ }^{\text {r,t }}$, A. Weber ${ }^{\mathrm{a}}$, R. Weill ${ }^{v}$, C. Willmott ${ }^{y}$, F. Wittgenstein ${ }^{\mathrm{q}}$, D. Wright ${ }^{\text {ai }}$, S.X. Wu ${ }^{\mathrm{r}}$, S. Wynhoff ${ }^{\mathrm{a}}$, Z.Z. Xu ${ }^{\mathrm{t}}$, B.Z. Yang ${ }^{t}$, C.G. Yang ${ }^{f}$, G. Yang ${ }^{r}$, X.Y. Yao ${ }^{f}$, C.H. Ye ${ }^{r}$, J.B. Ye ${ }^{t}$, Q. Ye ${ }^{r}$, S.C. Yeh ${ }^{\text {av }}$, J.M. You ${ }^{\mathrm{r}}$, N. Yunus ${ }^{\mathrm{r}}$, M. Yzerman ${ }^{\mathrm{b}}$, C. Zaccardelli af ${ }^{\text {, P. Zemp }}{ }^{\text {at }}$, M. Zeng ${ }^{\mathrm{r}}$, Y. Zeng ${ }^{\text {a }}$, D.H. Zhang ${ }^{\text {b }}$, Z.P. Zhang ${ }^{\text {t,r }}$, B. Zhou ${ }^{\text {j, G.J. Zhou }}{ }^{\text {f }, ~ J . F . ~ Z h o u ~}{ }^{\text {a }}$, R.Y. Zhu ${ }^{\text {af }}$, A. Zichichi ${ }^{\text {h,q,r, }}$, B.C.C. van der Zwaan ${ }^{b}$

"I. Physikalisches Institut, RWTH, D-52056 Aachen, FRG'

III. Physikalisches Institut. RWTH. D-52056 Aachen. FRG ${ }^{1}$

' National Institute for High Energy Physics. NIKHEF, NL-1009 DB Amsterdam. The Netherlands

" University of Michigan, Ann Arbor, MI 48109, USA

¿ Laboratoire d'Annecy-le-Vieux de Physique des Purticules, LAPP,IN2P3-CNRS, BP 110, F-7494I Annecy-le-Vieux CEDEX, France

¿ Johns Hopkins University. Baltimore. MD 21218, USA

institute of High Energy Physics. IHEP, 100039 Beijing, China

" Humboldt University. D-10099 Berlin, FRG ${ }^{1}$

" INFN-Sezione di Bologna, I-40/26 Bologna, Italy

'Tata Institute of Fundamental Research, Bombay 400005 . India

Boston University. Boston. MA 02215, USA

${ }^{k}$ Northeastern University, Boston, MA 02115, USA

Institute of Atomic Physics and University of Bucharest. R-76900 Bucharest, Romania

m Central Research Institute for Pliysics of the Hungarian Academy of Sciences, H-1525 Budapest 114, Hungary ${ }^{2}$

" Harvard University. Cambridge, MA 02139, USA

"Massuchusents Institute of Technology, Cambridge, MA 02139, USA

P INFN Sezione di Firenze and University of Florence, 1-50125 Florence, Italy

q European Laboratory for Particle Physics, CERN. CH-1211 Geneva 23, Switzerland

' World Luboratory. FBLJA Project, CH-1211 Geneva 23, Swizerland

"University of Geneva. CH-1211 Geneva 4, Switzerland

${ }^{t}$ Chinese University of Srience and Technology, USTC, Hefei, Anhui 230 029. China

"SEFT. Research Institute for High Energy Physics. P.O. Box 9, SF-00014 Helsinki, Finland

"University of Lausanne, CH-1015 Lausanne, Switzerland

"Los Alamos National Laboratory. Los Alamos. NM 87544, USA

* Institut de Physique Nucléaire de Luon. IN2P3-CNRS, Université Claude Bernard, F-69622 Villeurbanne Cedex. France

y Centro de Investigaciones Energeficas. Medioambientales y Tecnologicas, CIEMAT, E-28040 Madrid, Spain

'INFN-Sezione di Milano, I-20133 Milan. Italy

aa Institute of Theoretical and Experimental Physics, ITEP, Moscow, Russia

ah INFN-Sezione di Napoli and University of Naples, I-80/25 Naples, Italy

* Department of Natural Sciences. University of Cyprus. Nicosia. Cyprus

ad University of Nymegen and NIKHEF. NL-6525 ED Nymegen, The Netherlands

it Oak Ridge National Laboratory. Oak Ridge, TN 37831. USA

at Californiat Institute of Technology. Pasadena, CA 91125, USA

ag INFN-Sezione di Perugia and Universitá Degli Studi di Perugia, I-06100 Perugia, Italy

ath Carnegie Mellon University. Pittsburgh. PA 15213. USA

ai Princeton University, Princeton, NJ 08544, USA

aj INFN-Sezione di Roma and University of Rome. "La Sapienza", I-00185 Rome, Italy

ik Nuclear Plysics Institute, St. Petersburg, Russia

at University of California. San Diego, CA 92093, USA

am Dept. de Fisica de Particulas Elementales, Univ. de Santiago, E-15706 Santiago de Compostela. Spain

an Bulgarian Acudemy of Sciences, Institute of Mechatronics, BU-1113 Sofia, Bulgaria

an Center for High Energy Physics. Korea Advanced Inst. of Sciences and Technology, 305-701 Taejon. South Korea

ap University of Alabama, Tuscaloosa, AL 35486, USA

ay Purdue University, West Lafayette, IN 47907, USA

ar Paul Scherrer Institut. PSI, CH-5232 Villigen, Switzerland 


\author{
as DESY-Insfitut für Hochenergiephysik, D-15738 Zeuthen, FRG \\ at Eidgenössische Technische Hochschule, ETH Zürich, CH-8093 Zürich, Switzerland \\ an University of Hamburg, 2276I Hamburg, FRG \\ av High Energy Physics Group, Taiwan, ROC \\ Received 16 December 1994 \\ Editor: K. Winter
}

\begin{abstract}
We describe the sample of energetic single-photon events $\left(E_{\gamma}>15 \mathrm{GeV}\right)$ collected by L3 in the $1991-1993 \mathrm{LEP}$ runs. The event distributions agree with expectations from the Standard Model. The data are used to constrain the $\mathrm{ZZ} \gamma$ coupling and to set an upper limit of $4.1 \times 10^{-6} \mu_{B}(90 \%$ C.L.) on the the magnetic moment of the $\tau$ neutrino.
\end{abstract}

\section{Introduction}

The study of events produced in electron-positron collisions at the $Z$ resonance, in which the only finalstate particle detected is a photon, is sensitive to a variety of new physics processes. New processes contributing to the invisible width $\Gamma_{\mathrm{inv}}$ of the $\mathrm{Z}$ may be detected by counting single-photon events which arise from $\mathrm{Z}$ decay into stable, weakly interacting particles accompanied by a photon from initial-state radiation $[1,2]$. For center-of-mass energies near the $\mathrm{Z}$ resonance, the energy carried by photons from initial-state radiation tends to be a few $\mathrm{GeV}$ or less. A number of new physics models, e.g. supersymmetric models and compositeness models, also predict single-photon events in which the photon couples directly to the $Z$ or is produced by a radiative transition in the final state [3-8]. In contrast to $Z$ decay into invisible particles accompanied by a photon from initial-state radiation, the energy carried by these photons is typically a sizable fraction of the beam energy over a large region of the model parameter space.

We report below on the search for energetic singlephoton events $\left(E_{\gamma}>15 \mathrm{GeV}\right)$ in the data collected by L3 at LEP in 1991-1993. The energelic single-photon candidates are described in terms of their distributions

\footnotetext{
'Supported by the German Bundesministerium für Forschung und Technologie.

${ }^{2}$ Supported by the Hungarian OTKA fund under contract number 2970.

${ }^{3}$ Also supported by CONICET and Universidad Nacional de La Plata, CC 67, 1900 La Plata, Argentina.

${ }^{4}$ Deceased.
}

in energy and polar angle and compared with expectations from Standard Model processes. We interpret our data in terms of the $\tau$ neutrino magnetic moment and the $\mathrm{ZZ} \gamma$ coupling. Limits on new physics processes from energetic single-photon searches in data collected by L3 in 1990 and 1991 have been published previously $[9,10]$.

\section{The $\mathrm{L} 3$ detector}

The L3 detector is described in detail in [11]. Central tracking is performed by a Time Expansion Chamber (TEC) consisting of two coaxial cylindrical drift chambers with 12 inner and 24 outer sectors. The electromagnetic calorimeter is composed of bismuth germanate (BGO) crystals with coverage in polar angle from $11.4^{\circ}$ to $168.6^{\circ}$ except for gaps spanning $35.2^{\circ}-42.3^{\circ}$ and $137.7^{\circ}-144.8^{\circ}$. Hadronic energy depositions are measured by a uranium-proportional wire chamber sampling calorimeter (HCAL) surrounding the BGO and extending to within $6^{\circ}$ of the beamline. Active lead rings (ALR) of lead-scintillator sandwich construction are used to detect photons and electrons between 4.5 and 8.0 degrees from the beamline. Scintillator timing counters are located between the electromagnetic and hadronic calorimeters. The muon spectrometer, located outside the hadron calorimeter, comprises three layers of drift chambers measuring the muon trajectory in both the bending $(r-\phi)$ and nonbending $(z)$ planes. These subdetectors are installed inside a large magnet which provides a uniform field of 0.5 Tesla. BGO arrays mounted on either side of the 
detector are used for luminosity measurement. Their polar angle coverage is between $1.5^{\circ}$ and $3.9^{\circ}$ with respect to the beamline.

\section{Event selection}

Event selection was carried out on the data sample collected by L3 during the 1991-1993 LEP runs. The L3 detector triggered on energetic single-photon events using the logical OR combination of the BGO energy triggers, described in detail in [12].

The experimental signature is an energetic, electromagnetic shower in the $\mathrm{BGO}$ and an otherwise "empty" detector as defined below. In addition to possible new physics processes, events with this signature can occur due to (a) neutrino pair production accompanied by initial-state radiation, (b) QED events, e.g. $\mathrm{e}^{+} \mathrm{e}^{-} \rightarrow \mathrm{e}^{+} \mathrm{e}^{-} \gamma$, in which all final-state particles but the photon are outside the active volume of the detector, and (c) out-of-time cosmics. The number of events from process (a) can be reduced by taking advantage of the fact that initial-state radiation tends to be emitted along the beam direction and/or has energy which is typically of the order of $\Gamma_{Z}$. Events from process (b) can be eliminated by requiring the photon energy and production angle to be large enough so that by momentum conservation at least one other final-state particle is wcll within the active detector volume. Applying cuts on the shape of the shower is effective for reducing the contribution from cosmics. In order to suppress contributions from processes (a)(c) while retaining good acceptance for new physics processes, the following requirements were applied to the most energetic cluster found in the BGO:

- The energy of the BGO cluster must be greater than $15 \mathrm{GeV}$ and its polar angle must lie in the range $20^{\circ}<\theta<160^{\circ}$ (excluding the gap regions $34.5^{\circ}$ $<\theta<44.5^{\circ}$ and $135.5^{\circ}<\theta<145.5^{\circ}$ ).

- The transverse shape of the cluster must be consistent with a photon originating from the interaction point.

Apart from the energetic BGO cluster selected by the above cuts, the detector was required to be "empty" as defined by the following criteria. There must be no additional BGO clusters present comprised of 3 or more crystals with the most energetic crystal deposit exceeding $100 \mathrm{MeV}$. The energy detected in the other calorimeters must be attributable to noise or shower leakage from the BGO. There must be no tracks in the TEC and not more than one layer of the muon chamber containing reconstructed segments. The position and timing of the scintillator hits must be consistent with shower leakage or random noise. The "empty" detector cuts rejected beam-gas interactions, hadronic and charged leptonic decays of the Z, and QED events with two or more final-state particles within the acceptance. Cosmics were further suppressed by the cuts involving the scintillator counters and muon chambers.

We tuned the selection and evaluated its efficiency using Monte Carlo, random trigger events, and largeangle $\mathrm{e}^{+} \mathrm{e}^{-} \rightarrow \mathrm{e}^{+} \mathrm{e}^{-}$events. The trigger efficiency was measured by simulation following a procedure similar to the one used to measure our trigger efficiency for low-energy single-photon events [13]. The average trigger and selection efficiency combined was found to be $83 \pm 2 \%$ for those single-photon events passing the fiducial cuts on energy and angle listed above for the energetic BGO cluster. Of the total $17 \%$ inefficiency, $5 \%$ is due to the trigger, $4 \%$ to the "empty" detector cuts vetoing the event because of noise, and the remainder mainly to cuts on the shower shape, additional clusters in the BGO, and tracks in the TEC. The efficiency is independent of photon energy for the range of interest and is constant to within $\pm 5 \%$ in polar angle. In terms of equivalent integrated luminosity collected at the peak of the $\mathrm{Z}$ resonance, the data sample on which we conducted our search corresponds to $50.8 \mathrm{ph}^{-1}$. (The equivalent integrated luminosity at the peak is the sum of integrated luminosities collected at center-of-mass energies between 88.5 and $93.8 \mathrm{GeV}$, weighted by $\sigma_{\sqrt{s}}\left(e^{+} e^{-} \rightarrow\right.$ hadrons $) / \sigma_{\text {peak }}\left(e^{+} e^{-} \rightarrow\right.$ hadrons).)

A total of 9 events were found by our selection. The distributions of the photon energy and the cosine of its polar angle are shown in Fig. 1. Also shown are the Standard Model expectations from production of neutrino pairs accompanied by initial-state radiation, radiative Bhabha events, and annihilation into two photons accompanied by initial-state radiation. (The latter two processes contribute only in the data recorded before the installation of the ALR, corresponding to about $20 \%$ of the total data sample.) Background due to cosmics is negligible. The observed distributions are consistent with Standard Model predictions. The total number of events expected from the Standard Model 

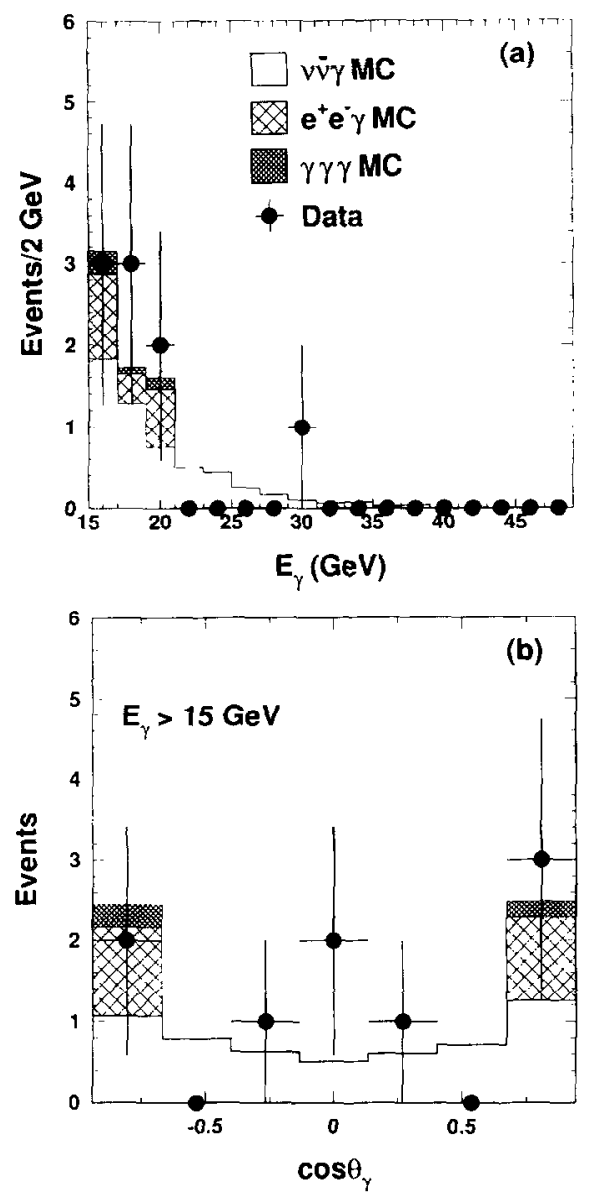

Fig. 1. (a) Distribution in energy of single-photon candidate events together with expectations from Standard Model processes. (b) The $\cos \theta_{y}$ spectrum of the single-photon candidates.

is 8.2 . If one instead requires that the photon energy be greater than half the beam energy, 1 event $\left(E_{\gamma}=\right.$ $30.2 \mathrm{GeV}, \cos \theta_{\gamma}=-0.32$ ) is selected from the data and 1.2 events are expected from the Standard Model in the $\nu \bar{\nu} \gamma$ channel.

\section{Constraints on the $\nu_{\tau}$ magnetic moment and $\mathrm{ZZ} \gamma$ coupling}

\section{1. $\nu_{\tau}$ magnetic moment}

The magnetic moment, $\mu_{\nu}$, of the $\tau$ neutrino is important for understanding the neutrino's basic nature and also for its relevance to issues such as whether or not a massive $\tau$ neutrino could be an important com-

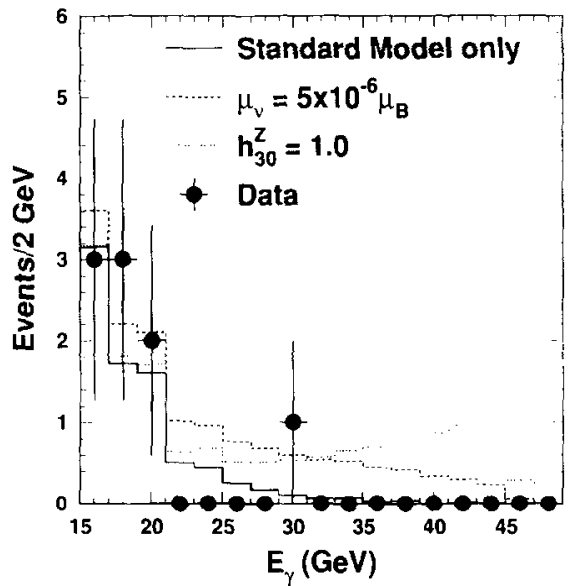

Fig. 2. The energy spectra of single-photon events expected in our search from (a) the Standard Model only (solid histogram), (b) the Standard Model modified to give the $\tau$ neutrino a magnetic moment of the magnitude indicated (dashed histogram), and (c) the Standard Model extended to include an anomalous ZZ $\gamma$ coupling (dotted histogram). The points show the energy spectrum of the single-photon candidates found in the search.

ponent of dark matter $[14,8,15]$. The production of single-photon events at $\mathrm{e}^{+} \mathrm{e}^{-}$colliders is sensitive to $\mu_{v}[8,15,16]$. At LEP energies, the dominant mechanism for the production of single-photon events via the magnetic moment interaction of the $\tau$ neutrino is radiation of a photon from the final-state neutrino or anti-neutrino. The dashed histogram in Fig. 2 indicates the energy distribution of single-photon events that would be expected if $\mu_{\nu}$ were equal to $5 \times 10^{-6} \mu_{B}$ where $\mu_{B}$ is the Bohr magneton. Since the photon is on-shell, the production rate depends on the magnetic moment form factor at $q^{2}=0$. We consider only the $\tau$ neutrino here because more stringent experimental upper bounds on the magnetic moments of the electron and muon neutrinos already exist [17].

To derive an upper limit on the magnetic moment, we assumed that for high photon energies the contributions due to the magnetic moment interaction and to the production of neutrino pairs accompanied by initial-state radiation do not interfere appreciably. Under this assumption, the additional production of single-photon events due to a $\nu_{\tau}$ magnetic moment varies as $\mu_{\nu}^{2}$. Starting from the differential Born cross section given in [16], we calculated the number of events expected as a function of $\mu_{\nu}$, taking into account the center-of-mass energy, the geometric accep- 
tance of our selection cuts, the combined trigger and sclection cfficiency, and initial-state radiation. The upper limit on the excess number of events allowed by our data was determined from Poisson statistics for the observed number of events and the expected Standard Model background. Requiring the photon energy to be greater than one half the beam energy, we obtain the limit

$\mu_{y^{\prime}}<4.1 \times 10^{-6} \mu_{B}$

at the $90 \%$ C.L.

The above bound applies to both static and transition magnetic moments. It is comparable with the bound of $4 \times 10^{-6} \mu_{B}$ (90\% C.L.) from low-energy experiments [15] and $3.4 \times 10^{-6} \mu_{B}$ (90\% C.I..) ${ }^{5}$ from the invisible width of the $Z$; with respect to these bounds it is unique in being a direct limit on the magnetic moment at $q^{2}=0$. The above bound is an order of magnitude weaker than that derived from a beam-dump experiment [19], but this limit requires assumptions on the $\mathrm{D}_{s}$ production cross section and its branching ratjo into $\tau \nu_{\tau}$, which are not yet measured.

\section{2. $Z Z \gamma$ coupling}

Energetic single-photon events are also sensitive to the $\mathrm{ZZ} \gamma$ coupling. The self-couplings of the electroweak gauge bosons are a prominent feature of the Standard Model. While WW $\gamma$ and WWZ couplings have received the most attention, the corresponding importance of searching experimentally for couplings between the neutral gauge bosons has also been noted $[5,20]$. Taking the ZZ $y$ coupling in particular, the most general vertex function invariant under Lorentz and electromagnetic gauge transformations can be described in terms of four independent dimensionless

\footnotetext{
${ }^{5}$ This limit was calculated in an approach similar to that taken in $\lfloor 18\rfloor$. The experimental value for the invisible width of the $Z$ was taken from Particle Data Group, Review of Particle Properties, Phys. Rev. D 50 (1994) 1173. The theoretical error on the $\mathrm{Z}$ invisible width was determined by running ZFITTER for the Standard Model with various choices of parameters. The mass of the top quark was varied according to a normal distribution centered at $174 \mathrm{GeV}$ with a $16 \mathrm{GeV}$ standard deviation. The Higgs mass was drawn from a box distribution between 60 and 1000 $\mathrm{GeV}$. The $\mathrm{Z}$ mass, $\alpha_{\mathrm{em}}$, and $\alpha_{s}$ were chosen from normal distributions with mean and standard deviations $91.187 \mathrm{GeV}, 0.007 \mathrm{GeV}$; $0.007819,0.000007$; and $0.123,0.006$, respectively. The theoretical and experimental errors were summed linearly.
}

form factors, denoted by $h_{i}^{Z}, i=1,2,3,4$. The contributions involving $h_{1}^{Z}$ and $h_{2}^{Z}$ are CP-violating while those involving the other pair of form factors are $\mathrm{CP}$ conserving. As is well known, all four form factors are zero at the tree level in the Standard Model. At the one-loop level, $h_{1}^{Z}$ and $h_{2}^{Z}$ are zero while the CPconserving form factors are nonzero but too small (e.g. $h_{3}^{Z} \approx 10^{-4}$ ) to lead to observable effects at any present or planned experiment. Thus observation of a $\mathrm{ZZ} \gamma$ coupling would signal physics beyond the Standard Model.

For $\mathrm{e}^{+} \mathrm{e}^{-}$annihilations at the $\mathrm{Z}$ resonance, the single-photon event topology is obtained for the case in which the photon is real and the final-state $Z$ decays into neutrinos. The $Z Z \gamma$ coupling would be manifest in the photon energy spectrum as an enhancement which becomes visible at $E_{\gamma} \sim 15 \mathrm{GeV}$ and increases monotonically with energy until near the kinematic limit. This is illustrated by the dotted histogram plotted in Fig. 2 representing the case where $h_{3}^{Z} \sim 1$. In order to set limits on the form factors $h_{i}^{Z}$, we follow [21] in adopting the parameterization

$h_{i}^{Z}=h_{i 0}^{Z} /\left(1+\left(P^{2} / \Lambda_{Z}^{2}\right)\right)^{n_{i}}$

where $\mathbf{P}$ is the four-momentum of the initial-state $\mathrm{Z}$, and $h_{i 0}^{Z}, \Lambda_{Z}$ and $n_{i}$ are parameters. The parameter $h_{i 0}^{Z}$ is the strength of the coupling in the low-energy limit and $\Lambda_{Z}$ may be interpreted as the energy at which the dynamic properties of the new physics underlying the anomalous coupling become visible. For further definiteness, also following [21], we take $n_{1}=n_{3}=$ 3.0 and $n_{2}=n_{4}=4.0$. With this choice of exponents the terms proportional to $h_{10}^{Z}$ and $h_{30}^{Z}$ have the same high-energy behavior as those proportional to $h_{20}^{Z}$ and $h_{40}^{Z}$; it should also be noted that unitarity requires that $n_{1}, n_{3}>1.5$ and $n_{2}, n_{4}>2.5$ regardless of the choice of $h_{i 0}^{Z}$ and $\Lambda_{Z}$.

To obtain upper limits on the $Z Z \gamma$ coupling, events were generated for various combinations of $\mathrm{ZZ} \gamma$ form factors and passed through the detector simulation and analysis programs. (Initial-state radiation was accounted for in the event generation procedure.) The results were used to parameterize the number of events expected in terns of combinations of $h_{i 0}^{Z}$ for different choices of scale $\Lambda_{z}$. As in the case of $\tau$ neutrino magnetic moment, limits were obtained requiring the photon energy to be greater than half the beam energy. 


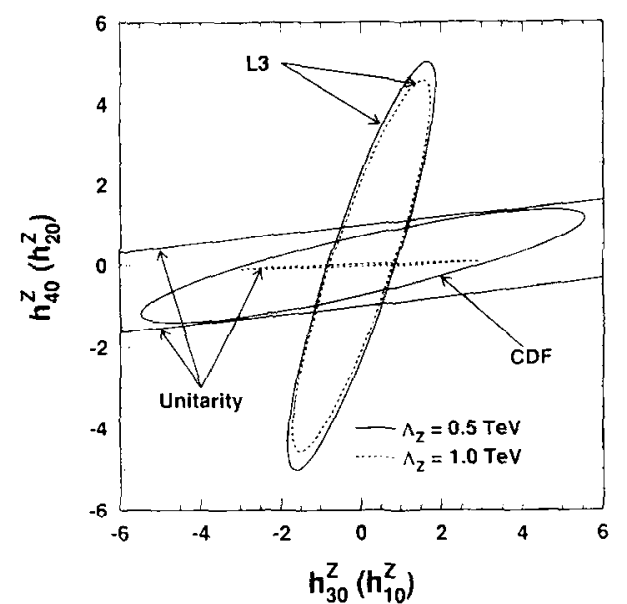

Fig. 3. Upper limits at the $95 \%$ C.L. on the $\mathrm{ZZ} \gamma$ coupling from L3 single-photon data for two different values of $\Lambda_{Z}$. The corresponding limits from unitarity are indicated. Also shown is the $95 \%$ upper limit contour for $\Lambda_{Z}=0.5 \mathrm{TeV}$ obtained by CDF [22] from study of the reaction $p \bar{p} \rightarrow \ell^{+} \ell^{-} \gamma X$.

Fig. 3 shows $95 \%$ C.L. limit contours from the L3 single-photon data in the $h_{30}^{Z}-h_{40}^{Z}\left(h_{10}^{Z}-h_{20}^{Z}\right)$ plane for scales $0.5 \mathrm{TeV}$ and $1.0 \mathrm{TeV}$. There is not much variation with scale, as expected if $\Lambda_{Z}$ is large compared to $m_{Z}$. Also displayed are the constraints imposed by the requirement that the cross sections respect unitarity at all energies. The unitarity limit is very sensitive to scale, decreasing as $\Lambda_{Z}^{-3}$ along the $h_{30}^{Z}$-axis and as $\Lambda_{Z}^{-4}$ along the $h_{40}^{Z}$-axis for our choice of $n_{i}$. The $\mathrm{CDF}$ collaboration has recently reported limits on the $\mathrm{ZZ} \gamma$ coupling [22] for an assumed scale of $0.5 \mathrm{TeV}$ obtained from study of the reaction $p \bar{p} \rightarrow \ell^{+} \ell^{-} \gamma X$. These are also plotted in Fig. 3. The L3 and CDF limit contours appear rotated with respect to each other because, as the effective center-of-mass energy varies from $m_{Z}$ (LEP) to the range of a few hundred $\mathrm{GeV}$ (Tevatron), the contributions to the amplitude from terms involving $h_{4}^{Z}\left(h_{2}^{Z}\right.$ ) rise steeply relative to those from terms involving $h_{3}^{Z}\left(h_{1}^{Z}\right)$.

To obtain the limits shown in Fig. 3, the form factor $h_{3}^{Z}\left(h_{1}^{Z}\right)$ has been assumed real relative to $h_{4}^{Z}\left(h_{2}^{Z}\right)$. In this case, the contributions from the two form factors interfere, as is evident from Fig. 3. On the other hand, the contributions of the form factors add incoherently if one member of the pair is chosen to be imaginary with respect to the other or if one member of the pair is CP-conserving and the other CP-violating. The L3 limit contours at the 95\% C.L. for pairs of non-interfering form factors can be determined from the axis intercepts in Fig. 3, which correspond to the limits on the associated form factor with all the other form factors assumed to be zero. For example, for $\Lambda_{Z}=0.5 \mathrm{TeV}$, the $h_{30}^{Z}$ axis intercepts are \pm 0.85 and the $h_{20}^{Z}$ axis intercepts are \pm 2.30 . Thus the limit contour in the $h_{20}^{Z}-h_{30}^{Z}$ plane would be an ellipse with major and minor axes parallel to the $h_{20}^{Z}$ and $h_{30}^{Z}$ axes, respectively, intercepting the $h_{20}^{Z}$ and $h_{30}^{Z}$ axes at \pm 2.30 and \pm 0.85 , respectively.

\section{Summary}

We have reported on the search for energetic singlephoton events in the data collected by L3 during 19911993 and have shown that the characteristics of the events found are consistent with what is predicted by the Standard Model. We have used the results to set an upper limit on the $\tau$ neutrino magnetic moment of $4.1 \times 10^{-6} \mu_{B}$ at the $90 \%$ C.L. and to constrain the $\mathrm{ZZ} \gamma$ coupling.

\section{Acknowledgments}

We are grateful to Ulrich Baur for providing a program to calculate $e^{+} e^{-} \rightarrow \nu \bar{\nu} \gamma$ cross sections in the presence of anomalous $\mathrm{Z} \gamma$ couplings. For allowing us to show their limits on the ZZy coupling prior to publication, we thank the $\mathrm{CDF}$ collaboration. We wish to express our gratitude to the CERN accelerator divisions for the excellent performance of the LEP machine. We acknowledge the contributions of all the engineers and technicians who have participated in the construction and maintenance of this experiment. Those of us who are not from member states thank CERN for its hospitality and help.

\section{References}

(1) A.D. Dolgov, L.B. Okun and V.I. Zakharov, Nucl. Phys. B 41 (1972) 197;

E. Ma and J. Okada, Phys. Rev. Lett. 41 (1978) 287;

K.J. Gaemers, R. Gastmans and F.M. Renard, Phys. Rev. D19 (1979) 1605:

G. Barbiellini, B. Richter and J. Siegrist, Phys. Lett. B 106 (1981) 414 
[2] ALEPH Collaboration, D. Buskulic et al., Phys. Lett. B 313 (1993) 520 ;

L3 Collaboration, O. Adriani et al., Phys, Lett. B 292 (1992) 463;

OPAL Collaboration, R. Akers et al., CERN preprint CERNPPE/94-105.

[3] M. Chen, C. Dionisi, M. Martinez and X. Tata, Phys. Rep. C 159 (1988) 203;

R. Barbieri et al., CERN-EP/89-08, Vol. 2 (1989) 121.

[4] F. Boudjema and A. Djouadi, Phys. Lett. B 240 (1990) 485.

[5] F.M. Renard, Nucl. Phys. B196 (1982) 93;

A. Barroso, F. Boudjema, J. Cole, and N. Dombey, Z. Phys. C28 (1985) 149.

[6] D.A. Dicus, S. Nandi, and J. Woodside, Phys. Lett. B 258 (1991) 231.

17\} J.E. Kim and U.W. Lee, Phys, Lett. B 233 (1989) 496.

[8] G.F. Giudice, Phys. Lett. B 251 (1990) 460.

[9] L3 Collaboration, B. Adeva et al., Phys. Lett. B 252 (1990) 525 ; L3 Collaboration, O. Adriani et al., Phys. Rep. 236 (1993) 1.

[10] L3 Collaboration, O. Adriani et al., Phys. Lett. B 297 (1992) 469.

The limit on $\beta$ given in this paper should be multiplied by a factor of two. It was obtained using an event generator based on the same program which was employed to calculate the numerical results reported in A. Barroso, P. Nogueira, and J.C. Romao, Phys. Lett. B 196 (1987) 547. This program contained an error which caused it to overestimate the effect of non-zero $\beta$ on the cross section by a factor of 4 (J.C. Romao, private communication). In terms of the notation used in the text, $\beta$ may be identified with $h_{30}^{Z}$ in the limit $\Lambda_{Z} \rightarrow \infty$.

[11] L3 Collaboration, B. Adeva et al., Nucl. Inst. Meth. A 289 (1990) 35

[12] R. Bizzarri et al., Nucl. Inst. Meth. A317 (1992) 463.

[13] L3 Collaboration, O. Adriani et al., Phys. Lett. B 292 (1992) 463.

[14] R.E. Shrock in Review of Particle Properties, Phys. Rev. D 50 (1994) 1385.

[15] H. Grotch and R.W. Robinett, Z. Phys. C 39 (1988) 553.

[16] T.M. Gould and I.Z. Rothstein, Phys. Lett. B 333 (1994) 545.

[17] X. Krakauer et al., Phys. Lett. B 252 (1990) 177.

[18] R. Escribano and E. Masso, Nucl. Phys, B429 (1994) 19.

[19] A.M. Cooper-Sarkar et al., Phys. Lett. B 280 (1992) 153

[20] D. Choudhury and S.D. Rindani, Phys. Lett. B 335 (1994) 198.

[21] U. Baur and E.L. Berger, Phys. Rev. D47 (1993) 4889.

[22] CDF Collaboration, F. Abe et al., Fermilab preprint PUB94/304-E, submitted to Phys. Rev. Lett.;

S. Errede in Proceedings of the XXVII International Conference on High Energy Physics, July 20-27, 1994, Glasgow, Scotland, in press;

see also CDF Collaboration, F. Abe et al., Fermilab preprint PUB-94/244-E, submitted to Phys. Rev. D. 